\title{
Well-posedness for a Class of Abstract Nonlinear Parabolic Systems with Time Delay*
}

\author{
H.T. Banks and C.J. Musante \\ Center for Research in Scientific Computation \\ and \\ Department of Mathematics \\ Box 8205 \\ North Carolina State University \\ Raleigh, NC 27695-8205
}

September 1997; revised December 31, 1997

\begin{abstract}
We consider a class of abstract nonlinear parabolic systems such as those arising in advanced toxicokinetic modeling. General well-posedness results in a weak or variational setting are given for systems without time delay. Once existence and uniqueness of solutions have been established, we extend the results to delay systems. The general theory is then applied to an explicit example, to establish wellposedness for a mathematical model describing the uptake and elimination of TCDD in humans.
\end{abstract}

Keywords and phrases: Nonlinear parabolic distributed parameter systems with delays, existence, uniqueness, variational form, toxicokinetic modeling, liver transport models.

\section{Introduction}

In this report we present existence, uniqueness, and continuous dependence results for a class of abstract nonlinear parabolic equations with time delay which arise in advanced toxicokinetic modeling. Our efforts were prompted by a mathematical model to describe the human uptake and elimination of $2,3,7,8$ tetrachlorodibenzo- $p$-dioxin (TCDD) [BMT]; in fact, these theoretical efforts are basic to our goal of developing computational methods for model verification, simulation and parameter estimation.

TCDD is considered to be the most toxic of a class of lipophilic chemicals [B] which exert their effects via interaction with an intracellular protein known as the aryl hydrocarbon (Ah) receptor. These chemicals are

*This work was supported in part by the Air Force Office of Scientific Research (AFOSR) under Grants F-49620-95-10236, F-49620-95-1-0375, and F-49620-93-1-0355. The research of C.J.M. was also supported by a US Department of Education Graduate Assistance in Areas of National Need (GAANN) Fellowship under Grant P200A50075, and the 1997 AFOSR Graduate Summer Research Program (GSRP) and the Armstrong Laboratory. 
of global social concern due to their presence and persistence in the environment and their ability to produce a wide range of toxic effects, including certain types of cancer [SDG, V], in laboratory animals. Moreover, TCDD was present in Agent Orange, the herbicide used by US forces in Vietnam.

The liver appears to be a major target organ for chronic toxicity of TCDD in rodents [SDG, V]. Physiologically based pharmaco- and toxicokinetic models which have attempted to describe the uptake, distribution, and elimination of chemicals in the body have generally used the "well-stirred" or "venous equilibrium" model to describe events occurring in the liver. The basic assumption of that model, that the concentration of solute is uniform throughout the liver, does not describe the elimination of solutes with decreasing concentration gradients along the liver acinus following a bolus input [RR1]. Moreover, the "well-stirred" model cannot accommodate spatial variations in biologically- and physiologically-based parameters, such as variations in enzyme activity and hepatic cell permeability. In 1985 a physiologically-based and spatially dependent dispersion model for hepatic elimination was introduced by Roberts and Rowland [RR1]. We show in [BMT] that this model can be adapted to describe the hepatic uptake and elimination of TCDD, incorporating the complex microcirculation of the human liver as well as TCDD interaction with two intracellular proteins: the non-inducible high-affinity, low capacity Ah receptor, and an inducible low-affinity, high-capacity microsomal protein, cytochrome P450 IA2 (CYP1A2) [PG, PTG, VA]. The model, which will be referred to as the TCDD model throughout this presentation, is "advanced" in that it includes spatial dispersion in the critical organ (the liver), time delays in tissue response, and nonlinear chemical kinetics within cellular compartments.

The organization of this paper is as follows. We begin in Section 2 with a brief discussion of the TCDD model. Motivated by this example, in Section 3 we present general results for abstract nonlinear parabolic systems with time delay. We address questions of well-posedness for the TCDD model in Section 4. Finally, Section 5 contains concluding remarks. In an appendix we give the particular equations for the TCDD model.

\section{The TCDD model}

A detailed description of the TCDD model is given in [BMT]. While an overview is presented here for the sake of completeness, the interested reader is referred to the original paper for in-depth model development.

In the TCDD model, transport of solute in the liver sinusoidal (blood) region is described by a convectiondispersion equation [RR1] to account for transport via bulk flow and turbulent diffusion. The general mass transport equation is then coupled with TCDD-specific equations, which include the kinetics of TCDDbinding to the Ah receptor protein, the induction of CYP1A2, and TCDD-binding to CYP1A2. The induction of CYP1A2 is modeled as a function of the fractional occupancy of the Ah receptor at a previous time to account for the many intracellular processes which must occur before this Ah receptor-mediated response is finally realized. Uptake of TCDD by the hepatocytes (liver cells) is modeled as passive diffusion across the cell membrane, with unbound TCDD the diffusing species. Metabolism, which is considered a detoxifying step for TCDD [SDG], is modeled as a first-order process.

Although data is not available on the time course of change of TCDD in the human liver, time-course data on serum TCDD levels in humans has been published [H]. Anticipating the use of this data in our numerical simulations, we coupled a "well-mixed" venous blood compartment to the liver model. The venous blood compartment includes a sink term to describe the rate of uptake of TCDD by adipose tissue, a primary TCDD storage site in the body [SDG, V]. The resultant mathematical model is a nonlinear system of seven 
coupled partial and ordinary differential equations with time delay.

To complete the system, boundary conditions for the TCDD model were formulated which yield a welldefined initial-boundary value problem. The exit boundary condition contains an unknown boundary term and thus must be estimated; that is, in the computational phase of our work observations of the solution will be used in an inverse algorithm to estimate this and possibly other unknown parameters.

Assuming an appropriate set of initial conditions, we thus are led to consider the initial-boundary value problem given in the Appendix with kinetic and rate constants as defined in [BMT].

\subsection{State space setting and problem formulation}

In this section we restate the initial-boundary value problem of (44) in terms of a weak or variational formulation. To simplify the analysis, we make a change of variable from $C_{B}$ to $\tilde{C}_{B}$, defined by

$$
\tilde{C}_{B}(t, z)=C_{B}(t, z)-C_{V b}(t),
$$

and note that $\tilde{C}_{B}$ satisfies a homogeneous Dirichlet boundary condition at $z=0$. We multiply the $i^{\text {th }}$ equation by a function $\phi_{i}$ in a "suitable" class of test functions and integrate in space in the first six equations, followed by integration by parts in the first equation (44a) only. Unless otherwise indicated, throughout the remainder of this section $\langle\cdot, \cdot\rangle$ will denote the usual $L_{2}$ inner product:

$$
\langle f, g\rangle=\int_{0}^{l} f(\xi) g(\xi) d \xi .
$$

As will readily be seen from our subsequent discussions, we may, without loss of generality, take all Hilbert spaces in our presentation to have real scalar fields.

Let $V=H_{L}^{1}(0, l)$ and $H=L_{2}(0, l)$, where

$$
H_{L}^{1}(0, l)=\left\{\phi \in H^{1}(0, l) \mid \phi(0)=0\right\},
$$

with $V$-norm

$$
|\phi|_{V}=\left|\phi^{\prime}\right|_{H}, \text { for all } \phi \in V .
$$

We define the state space $\mathcal{V}=V \times H^{5} \times \mathcal{R}$, and the space $\mathcal{H}=H^{6} \times \mathcal{R}$ with inner product

$$
\langle\phi, \psi\rangle_{\mathcal{H}}=\sum_{k=1}^{6}\left\langle\phi_{k}, \psi_{k}\right\rangle+\left\langle\phi_{7}, \psi_{7}\right\rangle_{\mathcal{R}} \quad \text { for all } \phi, \psi \in \mathcal{H}
$$

The weak or variational formulation of the problem (44) is as follows: we seek a solution $y(t) \in \mathcal{V}$ satisfying an appropriate initial condition $y(0)$ and

$$
\langle\dot{y}(t), \phi\rangle_{\mathcal{V}^{*}, \mathcal{V}}+\sigma(y(t), \phi)+\sigma_{D}\left(y\left(t-\tau_{c}\right), \phi\right)+\langle g(y(t)), \phi\rangle_{\mathcal{H}}+\left\langle g_{D}\left(y\left(t-\tau_{r}\right)\right), \phi\right\rangle_{\mathcal{H}}=\langle f(t), \phi\rangle_{\mathcal{V}^{*}, \mathcal{V}}
$$


for all $\phi \in \mathcal{V}$, where

$$
f(t)=\left(\begin{array}{c}
\dot{q}_{3}(t)-I(t)-a_{5} q_{2}(t) \delta_{l} \\
0 \\
0 \\
k_{s(A h)} \\
0 \\
k_{s(P r)} \\
I(t)-\dot{q}_{3}(t)
\end{array}\right), \quad g(y)=\left(\begin{array}{c}
0 \\
g_{A h}\left(y_{2}, y_{4}\right)+g_{P r}\left(y_{2}, y_{6}\right) \\
-g_{A h}\left(y_{2}, y_{4}\right) \\
g_{A h}\left(y_{2}, y_{4}\right) \\
-g_{P r}\left(y_{2}, y_{6}\right) \\
g_{P r}\left(y_{2}, y_{6}\right) \\
0
\end{array}\right), \quad g_{D}(y)=\left(\begin{array}{c}
0 \\
0 \\
0 \\
0 \\
0 \\
\left(\frac{I_{P r}}{V_{H}}\right) \frac{y_{3}}{y_{3}+y_{4}} \\
0
\end{array}\right),
$$

and the sesquilinear forms $\sigma, \sigma_{D}: \mathcal{V} \times \mathcal{V} \rightarrow \mathcal{R}$ are defined

$$
\begin{aligned}
\sigma(\psi, \phi)=\left\langle a_{1} \psi_{1}^{\prime}-a_{2} \psi_{1}, \phi_{1}^{\prime}\right\rangle+\left\langle a_{4} \psi_{1}-a_{3} \psi_{2}+\left(a_{4}-\alpha\right) \psi_{7}, \phi_{1}\right\rangle-\left\langle a_{2} \psi_{7} \delta_{l}, \phi_{1}\right\rangle_{V^{*}, V}+\left\langle k_{-1} \psi_{3}, \phi_{3}\right\rangle & \\
& +\left\langle b_{2} \psi_{2}-\left(b_{1} \psi_{1}+k_{-1} \psi_{3}+k_{-2} \psi_{5}+b_{1} \psi_{7}\right), \phi_{2}\right\rangle+\left\langle k_{d(A h)} \psi_{4}-k_{-1} \psi_{3}, \phi_{4}\right\rangle \\
& +\left\langle k_{-2} \psi_{5}, \phi_{5}\right\rangle+\left\langle k_{d(P r)} \psi_{6}-k_{-2} \psi_{5}, \phi_{6}\right\rangle+\left\langle\alpha \psi_{7}, \phi_{7}\right\rangle_{\mathcal{R}} \\
\sigma_{D}(\psi, \phi)=\alpha & \left.\alpha\left\langle\psi_{1}(l), \phi_{1}\right\rangle+\left\langle\psi_{7}, \phi_{1}\right\rangle-\left\langle\psi_{1}(l), \phi_{7}\right\rangle_{\mathcal{R}}-\left\langle\psi_{7}, \phi_{7}\right\rangle_{\mathcal{R}}\right] .
\end{aligned}
$$

Here, as in following sections, $\dot{y}=\frac{\partial y}{\partial t}, \phi^{\prime}=\frac{\partial \phi}{\partial z}$, and $\langle\cdot, \cdot\rangle_{V^{*}, V},\langle\cdot, \cdot\rangle_{\mathcal{V}^{*}, \mathcal{V}}$ denote the usual duality pairings as described below.

The system (1) motivated the theoretical developments presented in the following section, where we consider a general class of problems of which this example is a special case.

\section{Well-posedness for abstract nonlinear parabolic systems and systems with delay}

The arguments for existence and uniqueness presented in this section closely follow the work of Banks, Gilliam, and Shubov [BGS] for nonlinear hyperbolic systems and are repeated here with appropriate modification for nonlinear parabolic systems. After first establishing existence and uniqueness of solutions for a class of nonlinear parabolic systems on a finite time interval, we then show how these results can be extended to systems with delay.

\subsection{Formulation of the problem}

We seek to establish global existence of weak solutions for a class of abstract nonlinear parabolic systems of the form

$$
\begin{gathered}
\dot{y}(t)+\mathcal{A} y(t)+g(y(t))=F(t) \text { in } \mathcal{V}^{*} \\
y(0)=y_{0},
\end{gathered}
$$

for $t \in(0, T), T<\infty$. Throughout we assume there is a sequence of separable Hilbert spaces $\mathcal{V}, \mathcal{H}, \mathcal{V}^{*}$ forming a Gelfand triple [Wl] satisfying

$$
\mathcal{V} \hookrightarrow \mathcal{H} \simeq \mathcal{H}^{*} \hookrightarrow \mathcal{V}^{*}
$$


where we assume that the embedding $\mathcal{V} \hookrightarrow \mathcal{H}$ is dense and continuous with

$$
|\phi|_{\mathcal{H}} \leq k|\phi|_{\mathcal{V}} \text { for } \phi \in \mathcal{V} .
$$

We denote by $\langle\cdot, \cdot\rangle_{\mathcal{V} *}, \mathcal{V}$ the usual duality product [Wl, BSW], which is the extension by continuity of the inner product in $\mathcal{H}$, denoted $\langle\cdot, \cdot\rangle$ throughout the remainder of this section. The norm in $\mathcal{H}$ will be denoted $|\cdot|$. The operator $\mathcal{A}$ is defined (under the assumptions below) in terms of its sesquilinear form $\sigma: \mathcal{V} \times \mathcal{V} \rightarrow \mathcal{R}$; that is, $\mathcal{A} \in \mathcal{L}\left(\mathcal{V}, \mathcal{V}^{*}\right)$ and $\langle\mathcal{A} \phi, \psi\rangle_{\mathcal{V}}, \mathcal{V}=\sigma(\phi, \psi)$. We make the following standing assumptions:

A1) The form $\sigma$ is $\mathcal{V}$-bounded: for $\phi, \psi \in \mathcal{V}$

$$
|\sigma(\phi, \psi)| \leq c_{1}|\phi| \mathcal{V}|\psi|_{\mathcal{V}}
$$

A2) The form $\sigma$ is strictly coercive on $\mathcal{V}$ : for $\phi \in \mathcal{V}$

$$
\sigma(\phi, \phi) \geq k_{1}|\phi|_{\mathcal{V}}^{2}, \quad k_{1}>0 .
$$

A3) The forcing term $F$ satisfies

$$
F \in L_{2}\left((0, T), \mathcal{V}^{*}\right)
$$

A4) The nonlinear function $g$ is a continuous nonlinear mapping from $\mathcal{H}$ into $\mathcal{H}$ satisfying

$$
|g(\phi)| \leq \gamma|\phi|, \quad \phi \in \mathcal{H}
$$

for some positive constant $\gamma$.

A5) For any $\phi, \psi \in \mathcal{V}$,

$$
\langle g(\phi)-g(\psi), \phi-\psi\rangle+k_{1} k^{-1}|\phi-\psi|^{2} \geq 0
$$

where $k$ and $k_{1}$ are the constants in (3) and (5).

A6) For any $\phi \in \mathcal{H}$ the Fréchet derivative of $g$ exists and satisfies

$$
g^{\prime}(\phi) \in \mathcal{L}(\mathcal{H}, \mathcal{H}) \text { with }\left|g^{\prime}(\phi)\right|_{\mathcal{L}(\mathcal{H}, \mathcal{H})} \leq \tilde{C}_{3}
$$

Condition A6) will be useful in establishing uniqueness of solutions.

Given the above hypotheses and considerations, we consider the weak or variational form of the system given by

$$
\begin{aligned}
\langle\dot{y}(t), \phi\rangle+\sigma(y(t), \phi)+\langle g(y(t)), \phi\rangle & =\langle F(t), \phi\rangle \\
y(0) & =y_{0},
\end{aligned}
$$

for any $\phi \in \mathcal{V}$. We note that (2) and (10) are equivalent systems if we interpret the inner product $\langle\cdot, \cdot\rangle$ as $\langle\cdot, \cdot\rangle_{\mathcal{V} *}, \mathcal{\nu}$. 


\subsection{The main a priori estimate}

Choosing $\phi=y(t)$ in (10), we find that if a solution exists it must satisfy:

$$
\frac{1}{2} \frac{d}{d t}\left\{|y(t)|^{2}\right\}+\sigma(y(t), y(t))+\langle g(y(t)), y(t)\rangle=\langle F(t), y(t)\rangle_{\mathcal{V}^{*}, \mathcal{V}} .
$$

Integrating from 0 to $t$, for $t \in[0, T]$, and using condition (5) we obtain

$$
\frac{1}{2}|y(t)|^{2}+k_{1} \int_{0}^{t}\left\{|y(\xi)|_{\mathcal{V}}^{2}+\langle g(y(\xi)), y(\xi)\rangle\right\} d \xi \leq \frac{1}{2}|y(0)|^{2}+\int_{0}^{t}\langle F(\xi), y(\xi)\rangle_{\mathcal{L} *}, \mathcal{V} d \xi .
$$

Using the Cauchy-Schwarz inequality, the relation $2 a b \leq a^{2}+b^{2}$, and (7) we find

$$
|y(t)|^{2}+k_{1} \int_{0}^{t}|y(\xi)|_{\mathcal{V}}^{2} d \xi \leq\left|y_{0}\right|^{2}+\frac{1}{k_{1}} \int_{0}^{t}|F(\xi)|_{\mathcal{V}^{*}}^{2} d \xi+2 \gamma \int_{0}^{t}|y(\xi)|^{2} d \xi
$$

By ignoring the second term on the left of (12) and applying Gronwall's lemma we obtain

$$
|y(t)|^{2} \leq\left(\left|y_{0}\right|^{2}+\frac{1}{k_{1}} \int_{0}^{T}|F(\xi)|_{\mathcal{V}^{*}}^{2} d \xi\right) e^{2 \gamma t} .
$$

Next, substituting (13) back into (12) we have the desired a priori bound

$$
|y(t)|^{2}+k_{1} \int_{0}^{t}|y(\xi)|_{\mathcal{V}}^{2} \leq C
$$

where $C=C\left(\left|y_{0}\right|,|F|_{L_{2}\left((0, T), \mathcal{V}^{*}\right)}, \gamma, k_{1}\right)<\infty$ is constant.

\subsection{Galerkin approximations}

Let $\left\{\psi_{k}\right\}_{k=1}^{\infty} \subset \mathcal{V}$ be a linearly independent total subset of $\mathcal{V}$. We define the "Galerkin" approximations for (2a) by

$$
y^{N}(t)=\sum_{k=1}^{N} c_{k}^{N}(t) \psi_{k}
$$

where the coefficients $\left\{c_{k}^{N}(t)\right\}_{k=1}^{N}$ are chosen so that $y^{N}(t)$ is the unique solution of the $N$-dimensional system

$$
\left\langle\dot{y}^{N}(t), \psi_{j}\right\rangle+\sigma\left(y^{N}(t), \psi_{j}\right)+\left\langle g\left(y^{N}(t)\right), \psi_{j}\right\rangle=\left\langle F(t), \psi_{j}\right\rangle_{\mathcal{V}^{*}, \mathcal{V}}
$$

for $j=1, \ldots, N$, satisfying the initial condition

$$
c_{k}^{N}(0)=c_{0 k}^{N}
$$

The set $\left\{c_{0 k}^{N}\right\}$ is chosen such that

$$
y_{0}=\lim _{N \rightarrow \infty} \sum_{1}^{N} c_{0 k}^{N} \psi_{k} \text { in } \mathcal{H} .
$$


Multiplying (15) by $c_{j}^{N}(t)$ and summing over $j=1, \ldots, N$ we obtain (11) with $y$ replaced by $y^{N}$ :

$$
\frac{1}{2} \frac{d}{d t}\left\{\left|y^{N}(t)\right|^{2}\right\}+\sigma\left(y^{N}(t), y^{N}(t)\right)+\left\langle g\left(y^{N}(t)\right), y^{N}(t)\right\rangle=\left\langle F(t), y^{N}(t)\right\rangle_{\mathcal{V} *}, \mathcal{v} .
$$

Repeating the above arguments, we then obtain

$$
\left|y^{N}(t)\right|^{2}+k_{1} \int_{0}^{t}\left|y^{N}(\xi)\right|_{\mathcal{L}}^{2} d \xi \leq \tilde{C}
$$

where the constant $\tilde{C}$ is independent of $N$, depending on $y_{0}, F, \gamma$, and $k_{1}$ as in the constant $C$ of (14). We note that the convergence of $y_{0}^{N} \rightarrow y_{0}$ in $\mathcal{H}$ guarantees uniform boundedness of $\left|y_{0}^{N}\right|_{\mathcal{H}}$.

\subsection{Convergence of the Galerkin approximations}

To establish existence of solutions to (2) we will use the bounds of (16) to extract successive subsequences of the Galerkin approximations. We show that the final subsequence converges to a solution of (2). The subsequences selected at each step will be denoted by the same symbol $\left\{y^{N}\right\}$.

The following lemma is needed to carry out the proof of existence. The symbol $\stackrel{u}{\rightarrow}$ denotes weak convergence.

Lemma 1 There exists a subsequence $\left\{y^{N}\right\}$ of the original sequence of Galerkin approximations and $y \in$ $L_{2}((0, T), \mathcal{V})$ such that

a)

$$
y^{N} \stackrel{w}{-} y \text { in } L_{2}((0, T), \mathcal{V})
$$

b)

$$
y^{N}(t) \stackrel{u}{\longrightarrow} y(t) \text { in } \mathcal{H} \text { for each } t \in[0, T]
$$

c) There exists $h \in L_{2}((0, T), \mathcal{H})$ such that

$$
g\left(y^{N}\right) \stackrel{w}{\longrightarrow} h \text { in } L_{2}((0, T), \mathcal{H}) .
$$

Proof. The statements (17) and (18) follow from the fact that $\left\{y^{N}\right\}$ is bounded in $L_{2}((0, T), \mathcal{V})$ and $\left\{y^{N}(t)\right\}$ is bounded in $\mathcal{H}$ for each $t \in[0, T]$. Statement $c$ ) follows immediately from (3), (7), and (16).

\subsection{Existence of weak solutions}

In this section we obtain the fundamental existence result by letting $N \rightarrow \infty$ in an integral identity for the Galerkin approximations.

Theorem 1 Under assumptions A1)-A5), for $y_{0} \in \mathcal{H}$ there exists a weak solution $y$ of (2) with $y \in$ $L_{2}((0, T), \mathcal{V})$ and $\dot{y} \in L_{2}\left((0, T), \mathcal{V}^{*}\right)$. Furthermore, if condition $\left.\mathrm{A} 6\right)$ holds, the solution is unique. 
Proof. We denote by $P_{M}(M=1,2, \ldots)$ the class of functions $\eta \in L_{2}((0, T), \mathcal{V})$ which can be represented in the form

$$
\eta(t)=\sum_{k=1}^{M} a_{k}(t) \psi_{k},
$$

where $a_{k} \in C^{1}([0, T])$. Let

$$
P=\bigcup_{M=1}^{\infty} P_{M}
$$

The set $P$ is dense in $L_{2}((0, T), \mathcal{V})$. We multiply the $j^{\text {th }}$ equation in (15) by $a_{j}(t)$, take the sum from 1 to $M$ and integrate over $[0, t]$, and then integrate by parts in the first term to obtain

$$
\begin{gathered}
\int_{0}^{t}\left\{-\left\langle y^{N}(\xi), \dot{\eta}(\xi)\right\rangle+\sigma\left(y^{N}(\xi), \eta(\xi)\right)+\left\langle g\left(y^{N}(\xi)\right), \eta(\xi)\right\rangle\right\} d \xi+\left\langle y^{N}(t), \eta(t)\right\rangle \\
=\left\langle y^{N}(0), \eta(0)\right\rangle+\int_{0}^{t}\langle F(\xi), \eta(\xi)\rangle_{\mathcal{V}}, \mathcal{V} d \xi
\end{gathered}
$$

which is satisfied for all $\eta \in P_{M}, M \leq N$.

We fix $\eta \in P_{M}$ with $M \leq N$, and use (17), (18), and (19). Passing to the limit $N \rightarrow \infty$ in (22) we obtain

$$
\begin{aligned}
\int_{0}^{t}\{-\langle y(\xi), \dot{\eta}(\xi)\rangle & +\sigma(y(\xi), \eta(\xi))+\langle h(\xi), \eta(\xi)\rangle\} d \xi+\langle y(t), \eta(t)\rangle \\
& =\left\langle y_{0}, \eta(0)\right\rangle+\int_{0}^{t}\langle F(\xi), \eta(\xi)\rangle_{\mathcal{L} *, \mathcal{V}} d \xi
\end{aligned}
$$

Note all the statements of Lemma 1 are true for any interval $[0, t], t \leq T$. To pass to the limit in the first term under the integral sign we only need the weak convergence $y^{N} \stackrel{w}{\longrightarrow} y$ in $L_{2}((0, T), \mathcal{H})$ as in $(17)$. In the second term we note that for fixed $\eta \in \mathcal{V}$ the mapping $y \rightarrow \int_{0}^{t} \sigma(y(\xi), \eta(\xi)) d \xi$ is a bounded linear functional on $L_{2}((0, t), \mathcal{V})$ due to $(4)$, and therefore this functional is weakly continuous. This allows us to pass to the limit due to (17). In the third term we can pass to the limit due to (19). In the term outside the integral in the left side of (22) we can pass to the limit due to (18). We use the fact that $y^{N}(0) \rightarrow y_{0}$ strongly in $\mathcal{H}$ as $N \rightarrow \infty$ in the first term on the right hand side of (22).

Now for each $j$ choose $\eta_{j}(t)=a(t) \psi_{j}$ where we further restrict $a$ so that $a \in C_{0}^{\infty}[0, T]$. We obtain from (23)

$$
\int_{0}^{T} \dot{a}(\xi)\left\langle-y(\xi), \psi_{j}\right\rangle d \xi+\int_{0}^{T} a(\xi)\left\{\sigma\left(y(\xi), \psi_{j}\right)+\left\langle h(\xi), \psi_{j}\right\rangle-\left\langle F(\xi), \psi_{j}\right\rangle \mathcal{L}^{*}, \mathcal{V}\right\} d \xi=0
$$

This implies

$$
\left\langle\dot{y}(t), \psi_{j}\right\rangle+\sigma\left(y(t), \psi_{j}\right)+\left\langle h(t), \psi_{j}\right\rangle=\left\langle F(t), \psi_{j}\right\rangle_{\mathcal{L}^{*}, \mathcal{V}}
$$

for each $j$ and a.e. $t \in(0, T)$. Since $\left\{\psi_{j}\right\}$ is total in $\mathcal{V}$ we have $\dot{y} \in L_{2}\left((0, T), \mathcal{V}^{*}\right)$ and for all $\phi \in \mathcal{V}$

$$
\langle\dot{y}(t), \phi\rangle_{\mathcal{L}^{*}, \mathcal{V}}+\sigma(y(t), \phi)+\langle h(t), \phi\rangle=\langle F(t), \phi\rangle_{\mathcal{V}^{*}, \mathcal{V}} \quad \text { a.e. },
$$

which except for the term involving the limit function $h$ is the equation for weak solutions (10). We show first that $y(0)=y_{0}$ and then argue that the $h$ term under the integral of (23) can be replaced by $g(y)$ which yields that the limit function $y$ is a weak solution. 
To show $y(0)=y_{0}$ we return to Equation (23) which holds for each $\eta_{j}=a(t) \psi_{j}$, where $a \in C^{1}[0, T]$ and $a(0) \neq 0$. Integrating by parts in the first term in (23) and using (24) we obtain

$$
\langle y(t), \eta(t)\rangle+\langle-y(\xi), \eta(\xi)\rangle_{\xi=0}^{\xi=t}=\left\langle y_{0}, \eta(0)\right\rangle,
$$

or

$$
\left\langle y(0), \psi_{j}\right\rangle a(0)=\left\langle y_{0}, \psi_{j}\right\rangle a(0),
$$

for all $j$, from which it follows that $y(0)=y_{0}$.

To prove that the limit function $h$ is the correct term we use the Minty-Browder monotonicity method, as in [BGS].

Lemma 2 For any $\eta \in L_{2}((0, T), \mathcal{V})$ and $t \in[0, T]$,

$$
\int_{0}^{t}\langle g(y(\xi)), \eta(\xi)\rangle d \xi=\int_{0}^{t}\langle h(\xi), \eta(\xi)\rangle d \xi
$$

Proof. From (3) and (8) we obtain

$$
\int_{0}^{t}\left\{\langle g(u(\xi))-g(v(\xi)), u(\xi)-v(\xi)\rangle+k_{1}|u(\xi)-v(\xi)|_{\mathcal{V}}^{2}\right\} d \xi \geq 0
$$

for any $u, v \in L_{2}((0, T), \mathcal{V})$.

Select $u=y^{N} \in L_{2}((0, T), \mathcal{V})$ and any $v \in P^{M} \subset L_{2}((0, T), \mathcal{V})$ with $M \leq N$. Using condition (5) we obtain

$$
\int_{0}^{t}\left\langle g\left(y^{N}(\xi)\right)-g(v(\xi)), y^{N}(\xi)-v(\xi)\right\rangle d \xi+\int_{0}^{t} \sigma\left(y^{N}(\xi)-v(\xi), y^{N}(\xi)-v(\xi)\right) d \xi \geq 0 .
$$

Returning to (22), we take $\eta=y^{N}-v$ which is permissible since $y^{N} \in P^{N}$ and $v \in P^{M}, M \leq N$. This yields

$$
\begin{aligned}
\int_{0}^{t}\left\langle g\left(y^{N}(\xi)\right), y^{N}(\xi)-v(\xi)\right\rangle d \xi=\int_{0}^{t}\left\langle y^{N}(\xi), \dot{y}^{N}(\xi)-\dot{v}(\xi)\right\rangle d \xi-\int_{0}^{t} \sigma\left(y^{N}(\xi), y^{N}(\xi)-v(\xi)\right) d \xi \\
-\left\langle y^{N}(t), y^{N}(t)-v(t)\right\rangle+\left\langle y^{N}(0), y^{N}(0)-v(0)\right\rangle+\int_{0}^{t}\left\langle F(\xi), y^{N}(\xi)-v(\xi)\right\rangle_{\mathcal{L}^{*}, \mathcal{\nu}} d \xi .
\end{aligned}
$$

Now substituting (27) into (26) we obtain for all $v \in P_{M}, M \leq N$

$$
\begin{aligned}
-\frac{1}{2}\left|y^{N}(t)\right|^{2}+\frac{1}{2}\left|y^{N}(0)\right|^{2}+ & \int_{0}^{t}\left\{-\left\langle y^{N}(\xi), \dot{v}(\xi)\right\rangle-\sigma\left(v(\xi), y^{N}(\xi)-v(\xi)\right)-\left\langle g(v(\xi)), y^{N}(\xi)-v(\xi)\right\rangle\right\} d \xi \\
& +\int_{0}^{t}\left\langle F(\xi), y^{N}(\xi)-v(\xi)\right\rangle_{\mathcal{L}^{*}, \mathcal{V}} d \xi+\left\langle y^{N}(t), v(t)\right\rangle-\left\langle y^{N}(0), v(0)\right\rangle \geq 0 .
\end{aligned}
$$

We can pass to the limit $N \rightarrow \infty$ in (28) to obtain

$$
\begin{aligned}
-\frac{1}{2}|y(t)|^{2}+\frac{1}{2}\left|y_{0}\right|^{2}+\int_{0}^{t}\{-\langle y(\xi) & , \dot{v}(\xi)\rangle-\sigma(v(\xi), y(\xi)-v(\xi))-\langle g(v(\xi)), y(\xi)-v(\xi)\rangle\} d \xi \\
& +\int_{0}^{t}\langle F(\xi), y(\xi)-v(\xi)\rangle_{\mathcal{V}^{*}, \mathcal{V}} d \xi+\langle y(t), v(t)\rangle-\left\langle y_{0}, v(0)\right\rangle \geq 0 .
\end{aligned}
$$


We can pass to the limit in all terms under the integral sign due to the weak convergence results presented in Lemma 1. In the first term after the integral sign we can pass to the limit due to the weak convergence of $y^{N}(t) \stackrel{w}{\longrightarrow} y(t)$ in $\mathcal{H}$ for each $t \in[0, T]$. Strong convergence of $y^{N}(0) \rightarrow y_{0}$ in $\mathcal{H}$ allows us to pass to the limit in the second and last terms in the inequality. The first term in (28) requires further comment, as we only have weak convergence $y^{N}(t) \stackrel{w}{\longrightarrow} y(t)$ in $\mathcal{H}$ for any $t \in[0, T]$. However, since norms are weakly lower semicontinuous in Hilbert spaces,

$$
|y(t)|^{2} \leq \liminf _{N \rightarrow \infty}\left|y^{N}(t)\right|^{2}
$$

Therefore, we can pass to the limit in (28), obtaining the desired result (29). Note that (29) is valid for any $v \in P=\cup_{M=1}^{\infty} P^{M}$ and therefore for all $v \in L_{2}((0, T), \mathcal{V})$. Now we return to $(23)$ with $\eta=-v$ where $v$ is from (29):

$$
\int_{0}^{t}\{\langle y(\xi), \dot{v}(\xi)\rangle-\sigma(y(\xi), v(\xi))-\langle h(\xi), v(\xi)\rangle\} d \xi-\langle y(t), v(t)\rangle+\left\langle y_{0}, v(0)\right\rangle+\int_{0}^{t}\langle F(\xi), v(\xi)\rangle_{\mathcal{\nu} *}, \mathcal{\nu} \xi \xi=0
$$

Also, set $\eta=y$ in (23) to obtain

$$
\frac{1}{2}\left(|y(t)|^{2}-\left|y_{0}\right|^{2}\right)+\int_{0}^{t}\{\sigma(y(\xi), y(\xi))+\langle h(\xi), y(\xi)\rangle\} d \xi-\int_{0}^{t}\langle F(\xi), y(\xi)\rangle_{\mathcal{V}^{*}, \mathcal{V}} d \xi=0 .
$$

Adding inequality (29) and the relations (30) and (31), we find

$$
\int_{0}^{t}\{\sigma(y(\xi)-v(\xi), y(\xi)-v(\xi))+\langle h(\xi)-g(v(\xi)), y(\xi)-v(\xi)\rangle\} d \xi \geq 0 .
$$

For any $\theta>0$ and $\omega \in L_{2}((0, T), \mathcal{V})$, choose

$$
v(t)=y(t)-\theta \omega(t)
$$

Substituting (33) into (32) and dividing by $\theta>0$ we obtain

$$
\int_{0}^{t}\{\theta \sigma(\omega(\xi), \omega(\xi))+\langle h(\xi)-g(y(\xi)-\theta \omega(\xi)), \omega(\xi)\rangle\} d \xi \geq 0
$$

for any $\omega \in \mathcal{V}, \theta>0$. In (34) we can pass to the limit as $\theta \rightarrow 0$ and obtain the inequality

$$
\int_{0}^{t}\langle h(\xi)-g(y(\xi)), \omega(\xi)\rangle d \xi \geq 0
$$

using the condition that $g: \mathcal{H} \rightarrow \mathcal{H}$ is a continuous mapping.

The inequality $(35)$ holds for all $\omega \in L_{2}((0, T), \mathcal{V})$ only if it holds for equality. Therefore, Lemma 2 is established and the proof of existence is complete.

We now address the question of uniqueness of solutions. Let $w, v$ be two solutions of (10) corresponding to the initial condition $y_{0}$ and forcing term $F$. Note that $u \equiv w-v$ satisfies $u(0)=0$ and

$$
\langle u(t), \eta\rangle_{\mathcal{L}^{*}, \mathcal{V}}+\sigma(u(t), \eta)+\langle g(w(t))-g(v(t)), \eta\rangle=0 \text { for all } \eta \in \mathcal{V} .
$$

Observe $(36)$ as well as (10) holds for all $\eta \in L_{2}((0, T), \mathcal{V})$. For fixed $t \in[0, T]$ choose $\eta=u$ in $(36)$ and integrate from 0 to $t$ :

$$
\int_{0}^{t}\left\{\langle\dot{u}(\xi), u(\xi)\rangle_{\mathcal{V}^{*}, \mathcal{V}}+\sigma(u(\xi), u(\xi))+\langle\Delta g(\xi), u(\xi)\rangle\right\} d \xi=0
$$


where $\Delta g(\xi) \equiv g(w(\xi))-g(v(\xi))$. Since $\langle\dot{u}(\xi), u(\xi)\rangle=\frac{1}{2} \frac{d}{d \xi}\left\{|u(\xi)|^{2}\right\}$, this implies

$$
|u(t)|^{2}+2 \int_{0}^{t}\{\sigma(u(\xi), u(\xi))+\langle\Delta g(\xi), u(\xi)\rangle\} d \xi=0 .
$$

From condition (5) and (9) we obtain

$$
\begin{aligned}
|u(t)|^{2}+2 k_{1} \int_{0}^{t}|u(\xi)|_{\mathcal{V}}^{2} d \xi & \leq 2\left|\int_{0}^{t}\langle\Delta g(\xi), u(\xi)\rangle d \xi\right| \\
& \leq 2\left|\int_{0}^{t}\left\langle\int_{0}^{1} g^{\prime}(\theta w(\xi)+(1-\theta) v(\xi))[w(\xi)-v(\xi)] d \theta, u(\xi)\right\rangle d \xi\right| \\
& \leq 2 \int_{0}^{t} \tilde{C}_{3}|u(\xi)|^{2} d \xi
\end{aligned}
$$

Ignoring the second term in the inequality, we have for any $t \in[0, T]$

$$
|u(t)|^{2} \leq 2 \int_{0}^{t} \tilde{C}_{3}|u(\xi)|^{2} d \xi .
$$

Applying Gronwall's lemma, we find $u \equiv 0$ on any finite interval. Thus, the solution is unique and the proof of Theorem 1 is complete.

\subsection{Weakening the convexity condition on the nonlinearity}

In actual fact, for $y_{0} \in \mathcal{V}$ and $F=F_{1}+F_{2}$, where $F_{1} \in L_{2}((0, T), \mathcal{H})$ and $F_{2} \in H^{1}\left((0, T), \mathcal{V}^{*}\right)$, one can eliminate the "convexity condition" (8) on the nonlinearity by strengthening the convergence of the Galerkin approximations.

Returning to Section 3.3, we now choose the set $\left\{c_{0 k}^{N}\right\}$ such that

$$
y_{0}=\lim _{N \rightarrow \infty} \sum_{1}^{N} c_{0 k}^{N} \psi_{k} \text { in } \mathcal{V} .
$$

We then multiply (15) by $\dot{c}_{j}^{N}(t)$ and sum over $j=1, \ldots, N$ to obtain

$$
\left|\dot{y}^{N}(t)\right|^{2}+\frac{1}{2} \frac{d}{d t} \sigma\left(y^{N}(t), y^{N}(t)\right)+\left\langle g\left(y^{N}(t)\right), \dot{y}^{N}(t)\right\rangle=\left\langle F_{1}(t), \dot{y}^{N}(t)\right\rangle+\left\langle F_{2}(t), \dot{y}^{N}(t)\right\rangle_{\mathcal{L}^{*}, \mathcal{V}}
$$

We next integrate (37) from 0 to $t$ to obtain the equation

$$
\begin{aligned}
\int_{0}^{t}\left|\dot{y}^{N}(\xi)\right|^{2} d \xi+\frac{1}{2} \sigma\left(y^{N}(t), y^{N}(t)\right)+\int_{0}^{t}\left\langle g\left(y^{N}(\xi)\right), \dot{y}^{N}(\xi)\right\rangle d \xi \\
=\frac{1}{2} \sigma\left(y^{N}(0), y^{N}(0)\right)+\int_{0}^{t}\left[\left\langle F_{1}(\xi), \dot{y}^{N}(\xi)\right\rangle+\left\langle F_{2}(\xi), \dot{y}^{N}(\xi)\right\rangle_{\mathcal{L}^{*}, \mathcal{V}}\right] d \xi
\end{aligned}
$$

By conditions (4) and (5) we have

$$
\begin{gathered}
\frac{1}{2} k_{1}\left|y^{N}(t)\right|_{\mathcal{V}}^{2}+\int_{0}^{t}\left|\dot{y}^{N}(\xi)\right|^{2} d \xi \leq \frac{1}{2} c_{1}\left|y^{N}(0)\right|_{\mathcal{V}}^{2}+\int_{0}^{t}\left|\left\langle g\left(y^{N}(\xi)\right), \dot{y}^{N}(\xi)\right\rangle\right| d \xi+\int_{0}^{t}\left\langle F_{1}(\xi), \dot{y}^{N}(\xi)\right\rangle d \xi \\
+\int_{0}^{t}\left\langle F_{2}(\xi), \dot{y}^{N}(\xi)\right\rangle_{\mathcal{V}^{*}, \mathcal{V}} d \xi
\end{gathered}
$$


Considering the last term under the integral sign in (38), we note

$$
\left\langle F_{2}(t), \dot{y}^{N}(t)\right\rangle_{\mathcal{V}^{*}, \mathcal{V}}=\frac{d}{d t}\left\langle F_{2}(t), y^{N}(t)\right\rangle_{\mathcal{L}^{*}, \mathcal{v}}-\left\langle\dot{F}_{2}(t), y^{N}(t)\right\rangle_{\mathcal{V}^{*}, \mathcal{V}}
$$

Substituting (39) into (38) and taking absolute values we obtain the relation

$$
\begin{aligned}
\frac{k_{1}}{2}\left|y^{N}(t)\right|_{\mathcal{V}}^{2}+\int_{0}^{t}\left|\dot{y}^{N}(\xi)\right|^{2} d \xi \leq & \frac{c_{1}}{2}\left|y^{N}(0)\right|_{\mathcal{V}}^{2}+\int_{0}^{t}\left|\left\langle g\left(y^{N}(\xi)\right), \dot{y}^{N}(\xi)\right\rangle\right| d \xi+\int_{0}^{t}\left\langle F_{1}(t), \dot{y}^{N}(\xi)\right\rangle d \xi \\
& +\left\langle F_{2}(t), y^{N}(t)\right\rangle_{\mathcal{L}^{*}, \mathcal{V}}+\left|\left\langle F_{2}(0), y^{N}(0)\right\rangle_{\mathcal{V}^{*}, \mathcal{V}}\right|+\int_{0}^{t}\left|\left\langle\dot{F}_{2}(\xi), y^{N}(\xi)\right\rangle_{\mathcal{V}^{*}, \mathcal{V}}\right| d \xi
\end{aligned}
$$

Now by the Cauchy-Schwarz inequality, condition $(7)$, and the assumptions that $F_{1} \in L_{2}((0, T), \mathcal{H})$ and $F_{2} \in H^{1}\left((0, T), \mathcal{V}^{*}\right)$ we have for any $t \in[0, T]$,

$$
\begin{aligned}
\frac{k_{1}}{4}\left|y^{N}(t)\right|_{\mathcal{V}}^{2}+\frac{1}{4} \int_{0}^{t}\left|\dot{y}^{N}(\xi)\right|^{2} d \xi \leq & c_{1}\left|y^{N}(0)\right|_{\mathcal{V}}^{2}+\frac{\gamma^{2}}{2}\left|y^{N}\right|_{L_{2}((0, T), \mathcal{H})}^{2}+\left|F_{1}\right|_{L_{2}((0, T), \mathcal{H})}^{2} \\
& +\frac{1}{k_{1}}\left|F_{2}(t)\right|_{\mathcal{L}^{*}}^{2}+\frac{1}{2 c_{1}}\left|F_{2}(0)\right|_{\mathcal{L}^{*}}^{2}+\frac{1}{2}\left|\dot{F}_{2}\right|_{L_{2}\left((0, T), \mathcal{V}^{*}\right)}^{2}+\frac{1}{2}\left|y^{N}\right|_{L_{2}((0, T), \mathcal{V})}^{2},
\end{aligned}
$$

where we have repeatedly used the relation $2 a b \leq a^{2}+b^{2}$.

The convergence of $y^{N}(0) \rightarrow y_{0}$ strongly in $\mathcal{V}$ guarantees uniform boundedness of $\left|y^{N}(0)\right| \mathcal{V}$. The assumption $F_{2} \in H^{1}\left((0, T), \mathcal{V}^{*}\right)$ guarantees the existence of a constant $M$ such that $\left|F_{2}(t)\right|_{\mathcal{V}^{*}} \leq M$ for all $t \in[0, T]$. Furthermore, from $(16)$ we see that the set $\left\{y^{N}\right\}$ is bounded in $L_{2}((0, T), \mathcal{V}) \subset L_{2}((0, T), \mathcal{H})$ by a constant $\tilde{C}$. Therefore, we have shown

$$
\frac{k_{1}}{4}\left|y^{N}(t)\right|_{\mathcal{V}}^{2}+\frac{1}{4} \int_{0}^{t}\left|\dot{y}^{N}(\xi)\right|^{2} d \xi \leq \hat{C}
$$

where the constant $\hat{C}$ is independent of $N$ and $t$, depending only on $y_{0}, F_{1}, F_{2}, \gamma, k_{1}, c_{1}$ and $\tilde{C}$. Hence, the sets $\left\{y^{N}\right\}$ and $\left\{\dot{y}^{N}\right\}$ are bounded in $C([0, T], \mathcal{V})$ and $L_{2}((0, T), \mathcal{H})$, respectively.

Lemma 3 For $F=F_{1}+F_{2}$, where $F_{1} \in L_{2}((0, T), \mathcal{H})$ and $F_{2} \in H^{1}\left((0, T), \mathcal{V}^{*}\right)$, there exists a subsequence of the original sequence of Galerkin approximations such that

$$
\left\{y^{N}\right\} \rightarrow y \text { strongly in } C([0, T], \mathcal{H}) .
$$

Proof. We use the following version of the Arzela-Ascoli theorem [NS, Theorem 3.17.24]: If $Y$ is a complete metric space and $\mathcal{F} \subset C([0, T], Y)$, then $\mathcal{F}$ is relatively compact if and only if $\mathcal{F}$ is equicontinuous and $\{f(t): f \in \mathcal{F}\}$ is relatively compact in $Y$ for each $t \in[0, T]$.

Letting $Y=\mathcal{H}$ and $\mathcal{F}=\left\{y^{N}\right\} \subset C([0, T], \mathcal{H})$ in the statement of the theorem, one can show that the subset $\left\{y^{N}\right\}$ is equicontinuous in $C([0, T], \mathcal{H})$. Recall for each $t \in[0, T],\left\{y^{N}(t)\right\}$ is uniformly bounded in $\mathcal{V}$. Furthermore, since $\mathcal{V}$ embeds compactly in $\mathcal{H}$, for each $t \in[0, T]$ the set $\left\{y^{N}(t)\right\}$ is relatively compact in $\mathcal{H}$. Thus, the Arzela-Ascoli theorem allows us to conclude that $\left\{y^{N}\right\}$ is relatively compact in $C([0, T], \mathcal{H})$. Hence, there exists a subsequence of the original Galerkin approximations such that $y^{N} \rightarrow y$ strongly in $C([0, T], \mathcal{H})$. 
Theorem 2 Under the assumptions A1), A2) and A4) with $y_{0} \in \mathcal{V}$ and $F=F_{1}+F_{2}$, where $F_{1} \in$ $L_{2}((0, T), \mathcal{H}), F_{2} \in H^{1}\left((0, T), \mathcal{V}^{*}\right)$, there exists a weak solution $y$ of $(2)$ with $y \in L_{2}((0, T), \mathcal{V}) \cap C([0, T], \mathcal{H})$ and $\dot{y} \in L_{2}\left((0, T), \mathcal{V}^{*}\right)$. Furthermore, if condition $\left.\mathrm{A} 6\right)$ holds, the solution is unique and depends continuously on the data $\left(y_{0}, F\right)$.

Proof. For the existence argument, we need only to show that we can pass to the limit in (22). Lemma 3 and condition A4), that $g$ is a continuous mapping from $\mathcal{H}$ into $\mathcal{H}$, guarantee this result. Thus, (23) may be replaced by the equation

$$
\int_{0}^{t}\{-\langle y(\xi), \dot{\eta}(\xi)\rangle+\sigma(y(\xi), \eta(\xi))+\langle g(y(\xi)), \eta(\xi)\rangle\} d \xi+\langle y(t), \eta(t)\rangle=\left\langle y_{0}, \eta(0)\right\rangle+\int_{0}^{t}\langle F(\xi), \eta(\xi)\rangle_{\mathcal{L} * \mathcal{V}} d \xi
$$

Similar arguments to those in the proof of Theorem 1 follow directly from (40).

We now address the question of continuous dependence. Let $w, v$ be two solutions of (10), or equivalently (2), corresponding to the initial conditions $w_{0}, v_{0} \in \mathcal{V}$ and forcing function $F$. Note that $u \equiv w-v$ satisfies $|u(0)|=\left|w_{0}-v_{0}\right|$ and

$$
\langle u(t), \eta\rangle_{\mathcal{V}^{*}, \mathcal{V}}+\sigma(u(t), \eta)+\langle g(w(t))-g(v(t)), \eta\rangle=0
$$

for all $\eta \in \mathcal{V}$. Observe (41) as well as (10) holds for all $\eta \in L_{2}((0, T), \mathcal{V})$. For fixed $t \in[0, T]$ choose $\eta=u$ in (41) and integrate from 0 to $t$ :

$$
\int_{0}^{t}\left\{\langle\dot{u}(\xi), u(\xi)\rangle_{\mathcal{V}^{*}, \mathcal{V}}+\sigma(u(\xi), u(\xi))+\langle\Delta g(\xi), u(\xi)\rangle\right\} d \xi=0
$$

where $\Delta g(\xi) \equiv g(w(\xi))-g(v(\xi))$. Since $\langle\dot{u}(\xi), u(\xi)\rangle=\frac{1}{2} \frac{d}{d \xi}\left\{|u(\xi)|^{2}\right\}$, this implies

$$
|u(t)|^{2}+2 \int_{0}^{t}\{\sigma(u(\xi), u(\xi))+\langle\Delta g(\xi), u(\xi)\rangle\} d \xi=|u(0)|^{2} .
$$

From condition (5) and (9) we obtain

$$
\begin{aligned}
|u(t)|^{2}+2 k_{1} \int_{0}^{t}|u(\xi)|_{\mathcal{V}}^{2} d \xi & \leq|u(0)|^{2}+2\left|\int_{0}^{t}\langle\Delta g(\xi), u(\xi)\rangle d \xi\right| \\
& \leq k^{2}|u(0)|_{\mathcal{V}}^{2}+2\left|\int_{0}^{t}\left\langle\int_{0}^{1} g^{\prime}(\theta w(\xi)+(1-\theta) v(\xi))[w(\xi)-v(\xi)] d \theta, u(\xi)\right\rangle d \xi\right| \\
& \leq k^{2}|u(0)|_{\mathcal{V}}^{2}+2 \tilde{C}_{3} \int_{0}^{t}|u(\xi)|^{2} d \xi .
\end{aligned}
$$

Ignoring the second term in the inequality and applying Gronwall's lemma, we find

$$
|u(t)|^{2} \leq k^{2} e^{2 \tilde{C}_{3} T}|u(0)|_{\mathcal{V}}^{2}
$$

for all $t \in[0, T]$. Thus, we have established continuous dependence, in $C([0, T], \mathcal{H})$, on the initial condition. Continuous dependence on the forcing term $F$ follows by similar arguments. Thus, the proof of Theorem 2 is complete.

We have shown that for $y_{0} \in \mathcal{V}$ the convexity condition on the nonlinearity can be eliminated provided we have greater regularity on the forcing term $F$, either in space $\left(F \in L_{2}((0, T), \mathcal{H})\right)$ or time $\left(F \in H^{1}\left((0, T), \mathcal{V}^{*}\right)\right)$. 
This method of transferring smoothness in space to smoothness in time is analogous to that found in discussions as to when mild solutions provide classical solutions to inhomogeneous abstract Cauchy problems (see Pazy [P, Chapter 4.2]). We also note that if one takes $F_{2} \equiv 0$ in $H^{1}\left((0, T), \mathcal{V}^{*}\right)$, the case $F \in L_{2}((0, T), \mathcal{H})$ can be treated under the conditions of Theorem 2.

\subsection{Existence of weak solutions for systems with time delay}

This section is concerned with extending the result of the previous section to systems with delay. We consider

$$
\begin{gathered}
\dot{y}(t)+\mathcal{A} y(t)+\mathcal{A}_{D}\left(y\left(t-\tau_{1}\right)\right)+g(y(t))+g_{D}\left(y\left(t-\tau_{2}\right)\right)=F(t) \text { in } \mathcal{V}^{*} \\
y(s)=y_{0}(s), \quad s \in[-\tau, 0],
\end{gathered}
$$

where $\tau=\max \left\{\tau_{1}, \tau_{2}\right\}, 0<\tau<T$. For simplicity we take $\tau_{1}=\tau_{2}$ in the discussion that follows; similar arguments hold for the case of inequality. The linear operator $\mathcal{A}_{D}$ is defined in terms of its sesquilinear form $\sigma_{D}: \mathcal{V} \times \mathcal{V} \rightarrow \mathcal{R}$; that is, $\mathcal{A}_{D} \in \mathcal{L}\left(\mathcal{V}, \mathcal{V}^{*}\right)$ and $\left\langle\mathcal{A}_{D} \phi, \psi\right\rangle_{\mathcal{V}^{*}, \mathcal{V}}=\sigma_{D}(\phi, \psi)$. We assume the same setting as considered in Theorem 2, with A6) and the following additional conditions on the delay terms holding:

D6) The form $\sigma_{D}$ is $\mathcal{V}$-bounded: for $\phi, \psi \in \mathcal{V}$,

$$
\left|\sigma_{D}(\phi, \psi)\right| \leq c_{2}|\phi| \mathcal{V}|\psi|_{\mathcal{V}}
$$

D7) The function $g_{D}$ is a nonlinear mapping from $\mathcal{H}$ into $\mathcal{H}$.

Note that it is not necessary for the nonlinear term with delay, $g_{D}$, to be a continuous mapping.

Theorem 3 Under the assumptions above, there exists a unique weak solution $y$ of $(42)$ with $y \in L_{2}((0, T), \mathcal{V}) \cap$ $C([0, T], \mathcal{H})$ and $\dot{y} \in L_{2}\left((0, T), \mathcal{V}^{*}\right)$.

By Theorem 2 we have the existence of a unique solution $y \in L_{2}((0, \tau), \mathcal{V}) \cap C([0, \tau], \mathcal{H})$ of the system

$$
\begin{gathered}
\langle\dot{y}(t), \phi\rangle_{\mathcal{V}^{*}, \mathcal{V}}+\sigma(y(t), \phi)+\langle g(y(t)), \phi\rangle=\left\langle F(t)-G_{1}(t), \phi\right\rangle_{\mathcal{V}^{*}, \mathcal{V}}, \\
y(0)=y_{0}(0),
\end{gathered}
$$

where $G_{1} \in L_{2}\left((0, \tau), \mathcal{V}^{*}\right)$ is defined

$$
G_{1}(t)(\phi)=\left\langle\mathcal{A}_{D} y_{0}(t-\tau)+g_{D}\left(y_{0}(t-\tau)\right), \phi\right\rangle_{\mathcal{V} * \mathcal{V}}
$$

Now setting

$$
\Phi(t)=y(t-\tau)
$$

we observe that $\Phi(t) \in \mathcal{V}$ is a known function of time on $[\tau, 2 \tau]$. Defining $G_{2} \in L_{2}\left((\tau, 2 \tau), \mathcal{V}^{*}\right)$ by

$$
G_{2}(t)(\phi)=\left\langle\mathcal{A}_{D} \Phi(t)+g_{D}(\Phi(t)), \phi\right\rangle_{\mathcal{V} *}, \mathcal{V}
$$


for all $\phi \in \mathcal{V}$, we thus seek a solution $y$ on the interval $[\tau, 2 \tau]$ satisfying

$$
\begin{aligned}
\langle\dot{y}(t), \phi\rangle_{\mathcal{V}, \mathcal{V}}+\sigma(y(t), \phi)+\langle g(y(t)), \phi\rangle & =\left\langle F(t)-G_{2}(t), \phi\right\rangle_{\mathcal{V} *, \mathcal{V}} \\
y(\tau) & =\Phi(2 \tau),
\end{aligned}
$$

for all $\phi \in \mathcal{V}$. By Theorem 2 a solution $y$ of (43) exists and is unique. We thereby continue in this manner to "step" the solution forward by time intervals of length $\tau$ until the desired final time $T$ is achieved. This is the standard "method of steps" or "method of continuation" used in delay differential equations by which the solution is extended forward in time $[\mathrm{BC}]$.

\section{Well-posedness for the TCDD model}

In this section we apply the theoretical results of Section 3 to the TCDD model. We thus consider (1) and recall the definitions of $V, H$ and $\mathcal{V}, \mathcal{H}$ given in Section 2. Note $\mathcal{V}$ and $\mathcal{H}$ form the usual Gelfand triple as discussed in Section 3.1, with the usual duality pairing $\langle\cdot, \cdot\rangle_{\mathcal{V}}, \mathcal{V}$, where $\mathcal{V}^{*}=V^{*} \times H^{5} \times \mathcal{R}$.

We state the following without proof:

(H1) $\sigma$ is $\mathcal{V}$-continuous: There exists a constant $\gamma_{1}>0$ such that for all $\psi, \phi \in \mathcal{V}$

$$
|\sigma(\psi, \phi)| \leq \gamma_{1}|\psi|_{\mathcal{V}}|\phi|_{\mathcal{V}}
$$

(H2) $\sigma$ is $\mathcal{V}$-coercive: There exist constants $k_{1}>0, \lambda_{0}>0$ such that for all $\phi \in \mathcal{V}$

$$
\sigma(\phi, \phi) \geq k_{1}|\phi|_{\mathcal{V}}^{2}-\lambda_{0}|\phi|_{\mathcal{H}}^{2}
$$

(H3) $\sigma_{D}$ is $\mathcal{V}$-continuous: There exists a constant $\gamma_{2}>0$ such that for all $\psi, \phi \in \mathcal{V}$

$$
\left|\sigma_{D}(\psi, \phi)\right| \leq\left.\left.\gamma_{2}|\psi|_{\mathcal{V}}\right|_{\phi}\right|_{\mathcal{V}}
$$

Under the conditions (H1) and (H3) above, there exist continuous linear operators $\mathcal{A}, \mathcal{A}_{D}: \mathcal{V} \rightarrow \mathcal{V}^{*}$ such that $\sigma(\phi, \psi)=\langle\mathcal{A} \phi, \psi\rangle_{\mathcal{V}^{*}, \mathcal{V}}$ and $\sigma_{D}(\phi, \psi)=\left\langle\mathcal{A}_{D} \phi, \psi\right\rangle_{\mathcal{V}^{*}, \mathcal{V}}$ for all $\phi, \psi \in \mathcal{V}[\mathrm{Wl}]$. Define $F \in L_{2}\left((0, T), \mathcal{V}^{*}\right)$ by

$$
F(t)(\phi)=\langle f(t), \phi\rangle_{\mathcal{V} *}, \mathcal{V},
$$

where $f$ is as defined in Section 2.1. Under the hypotheses and conditions above, Equation (1) may equivalently be written in the operator-theoretic form

$$
\dot{y}(t)+\mathcal{A} y(t)+\mathcal{A}_{D} y\left(t-\tau_{c}\right)+g(y(t))+g_{D}\left(y\left(t-\tau_{r}\right)\right)=F(t) \text { in } \mathcal{V}^{*} .
$$

This is similar to the system in (42), however we observe by $\mathrm{H} 2$ ) that the sesquilinear form $\sigma$ is not strictly coercive; that is, condition A2) is not satisfied. However, as discussed in Wloka [Wl], this problem is easily resolved by a standard modification to the system.

We note that the nonlinear term $g$ is globally bounded in $\mathcal{H}$ and $g^{\prime}(\phi)$ is globally bounded in $\mathcal{L}(\mathcal{H}, \mathcal{H})$ for each $\phi \in \mathcal{H}$, however $g$ does not satisfy the convexity condition A5) of Theorem 1 . We showed in Theorem 2 that it is possible to remove the convexity condition on $g$ provided the forcing term $F$ has 
sufficient smoothness, either in time $\left(F \in H^{1}\left((0, T), \mathcal{V}^{*}\right)\right)$ or space $\left(F \in L_{2}((0, T), \mathcal{H})\right)$. For the TCDD model, the forcing term involves the pointwise evaluation of a test function at the right boundary; that is, $F$ involves a Dirac delta function, $\delta_{l}$, and therefore $F \notin L_{2}((0, T), \mathcal{H})$. However, by imposing reasonable and biologically plausible conditions on the smoothness of certain time-dependent parameters, $F$ can be written in terms of an $L_{2}((0, T), \mathcal{H})$ and $H^{1}\left((0, T), \mathcal{V}^{*}\right)$ component. Therefore, the hypotheses of Theorem 3 are satisfied and existence and uniqueness of the solution for the TCDD model is established.

\section{Concluding Remarks}

Motivated by a convection-dispersion model for the uptake and elimination of TCDD in humans, we have presented a general result for existence and uniqueness of solutions for a class of abstract nonlinear parabolic equations. We have shown that one condition pertaining to the nonlinearity, the so-called "convexity condition", can be relaxed provided one has greater regularity on the forcing term, either in space or in time. These results can be extended to a class of nonlinear parabolic systems with time delay by the "method of steps" used in the study of delay differential equations. Finally, arguments for the existence and uniqueness of a weak solution for the TCDD model are given.

Acknowledgments. C.J.M. would like to thank Dr. Richard A. Albanese, Mathematical Products Division, Armstrong Laboratory (AL/OES), Brooks AFB, TX, for his input and encouragement during her internship at AL/OES as part of the 1997 AFOSR Graduate Summer Research Program. 


\section{A The TCDD model system of equations}

The reader is referred to [BMT] for a detailed description of the model and its development, including definitions of rate constants and the nonlinear functions $g_{A h}, g_{P r}$. We note that $g_{A h}, g_{P r}$ are saturating nonlinearities modified from the usual product terms arising from the law of mass action in chemical kinetics (see $[\mathrm{BMT}]$ for detailed discussions) and thus are, along with their derivatives, bounded functions.

$$
\begin{aligned}
& \left(V_{B}+V_{D} \frac{f_{u_{B}}}{f_{u_{D}}}\right) \frac{\partial C_{B}}{\partial t}=V_{B} \mathcal{D} \frac{\partial^{2} C_{B}}{\partial z^{2}}-V_{B} v \frac{\partial C_{B}}{\partial z}+P\left(C_{u_{H}}-f_{u_{B}} C_{B}\right), \\
& \frac{\partial C_{u_{H}}}{\partial t}=\frac{P f_{u_{B}}}{V_{H}} C_{B}-\left(\frac{P}{V_{H}}+k_{3}\right) C_{u_{H}}-g_{A h}\left(C_{u_{H}}, C_{A h}\right) \\
& +k_{-1} C_{A h-T C D D}-g_{P r}\left(C_{u_{H}}, C_{P r}\right)+k_{-2} C_{P r-T C D D}, \\
& \frac{\partial C_{A h-T C D D}}{\partial t}=g_{A h}\left(C_{u_{H}}, C_{A h}\right)-k_{-1} C_{A h-T C D D}, \\
& \frac{\partial C_{A h}}{\partial t}=k_{-1} C_{A h-T C D D}-g_{A h}\left(C_{u_{H}}, C_{A h}\right)-k_{d(A h)} C_{A h}+k_{s(A h)}, \\
& \frac{\partial C_{P r-T C D D}}{\partial t}=g_{P r}\left(C_{u_{H}}, C_{P r}\right)-k_{-2} C_{P r-T C D D} \text {, } \\
& \frac{\partial C_{P r}}{\partial t}=k_{-2} C_{P r-T C D D}-g_{P r}\left(C_{u_{H}}, C_{P r}\right)-k_{d(P r)} C_{P r}+k_{s(P r)} \\
& +\left(\frac{I_{P r}}{V_{H}}\right) \frac{C_{A h-T C D D}\left(t-\tau_{r}, z\right)}{C_{A h}\left(t-\tau_{r}, z\right)+C_{A h-T C D D}\left(t-\tau_{r}, z\right)}, \\
& \frac{d C_{V b}}{d t}(t)=\frac{Q_{V b}}{V_{V b}}\left(C_{B}\left(t-\tau_{e}, l\right)-C_{V b}(t)\right)+I(t)-\dot{q}_{3}(t), \\
& C_{B}(t, 0)=C_{V b}(t), \\
& v C_{B}(t, l)-\mathcal{D} \frac{\partial C_{B}}{\partial z}(t, l)=q_{2}(t) \text {. }
\end{aligned}
$$




\section{References}

[B] L.S. Birnbaum, The mechanism of dioxin toxicity: relationship to risk assessment, Environ. Health Perspect. 102 157-167 (1994).

[BC] R. Bellman and K.L. Cooke, Differential-Difference Equations, Rand Corporation (1963).

[BGS] H.T. Banks, D.S. Gilliam, and V.L. Shubov, Global solvability for damped abstract nonlinear hyperbolic systems, Differential and Integral Equations 10 309-332 (1997).

[BIW] H.T. Banks, K. Ito, and Y. Wang, Well-posedness for damped second order systems with unbounded input operators, CRSC-TR93-10, June 1993, Differential and Integral Equations 8 587-606 (1995).

[BMT] H.T. Banks, C.J. Musante, and H.T. Tran, A dispersion model for the hepatic uptake and elimination of 2,3,7,8-tetrachlorodibenzo- $p$-dioxin, CRSC-TR97-29, September, 1997, Mathematical and Computer Modelling, submitted.

[BSW] H.T. Banks, R.C. Smith, and Y. Wang, Smart Material Structures: Modeling Estimation, and Control, J. Wiley \& Sons (1996).

[H] G.L. Henriksen, N.S. Ketchum, J.E. Michaelek, and J.A. Swaby, Serum dioxin and diabetes mellitus in veterans of Operation Ranch Hand, Epidemiology 8(3) 252-258 (1997).

[NS] A.W. Naylor and G.R. Sell, Linear Operator Theory in Engineering and Science, Springer-Verlag, New York (1982).

[P] A. Pazy, Semigroups of Linear Operators and Applications to Partial Differential Equations, SpringerVerlag, New York (1983).

[PG] A. Poland and E. Glover, Stereospecific, high affinity binding of 2,3,7,8-tetrachlorodibenzo- $p$-dioxin by hepatic cytosol, J. Biol. Chem. 251 4936-4946 (1976).

[PTG] A. Poland, P. Teitelbaum, and E. Glover, [ $\left.{ }^{125} \mathrm{I}\right]$ 2-Iodo-3,7,8-trichlorodibenzo- $p$-dioxin-binding species in mouse liver induced by agonists for the Ah receptor: characterization and identification, Mol. Pharmacol. 36 113-120 (1989).

[RR1] M.S. Roberts and M. Rowland, A dispersion model of hepatic elimination: 1. Formulation of the model and bolus considerations, J. Pharmacokinet. Biopharm. 14 227-260 (1986).

[SDG] S.A. Skene, I.C. Dewhurst, and M. Greenberg, Polychlorinated dibenzo- $p$-dioxins and polychlorinated dibenzofurans: the risks to human health, A review, Hum. Toxicol. 8 173-203 (1989).

[V] M. Van den Berg, J. De Jongh, H. Poiger, and J. Olson, The toxicokinetics and metabolism of polychlorinated dibenzo- $p$-dioxins (PCDDs) and dibenzofurans (PCDFs) and their relevance for toxicity, Crit. Rev. Toxicol. 24 1-74 (1994).

[VA] R. Voorman and S.D. Aust, Specific binding of polyhalogenated aromatic hydrocarbon inducers of cytochrome P-450d to the cytochrome and inhibition of its estradiol-2-hydroxylase activity, Toxicol. Appl. Pharmacol. 90 69-78 (1987).

[Wl] J. Wloka, Partial Differential Equations, Cambridge University Press (1992). 